Review

\title{
Role of myo-inositol phosphate synthase and sucrose synthase genes in plant seed development
}

\author{
Ghassen Abid ${ }^{\mathrm{a}, \mathrm{b}, *}$, Souleymane Silue ${ }^{\mathrm{a}}$, Yordan Muhovski ${ }^{\mathrm{b}}$, Jean-Marie Jacquemin ${ }^{\mathrm{b}}$, \\ André Toussaint ${ }^{a}$, Jean-Pierre Baudoin ${ }^{a}$ \\ a Unit of Tropical Crop Husbandry and Horticulture, Gembloux Agricultural University, Passage des Déportés 2, B-5030 Gembloux, Belgium \\ b Department of Biotechnology, Walloon Agricultural Research Centre, Chaussé de Charleroi, 234, B-5030 Gembloux, Belgium
}

\section{A R T I C L E I N F O}

\section{Article history:}

Received 4 August 2008

Received in revised form 7 March 2009

Accepted 11 March 2009

Available online 20 March 2009

Received by A.J. van Wijnen

\section{Keywords:}

myo-inositol phosphate synthase (MIPS)

Sucrose synthase (Sus)

Seed development

\begin{abstract}
A B S T R A C T
The aim of this review is to highlight the role of myo-inositol phosphate synthase (MIPS), which catalyses the first step in inositol biosynthesis and of sucrose synthase (Sus), an enzyme involved in UDP-glucose formation, the principal nucleoside diphosphate in the sucrose cleavage reaction and in trehalose biosynthesis. These two enzymes are involved in various physiological processes including seed growth and resistance to biotic and abiotic stresses.

The study of mutated MIPS and Sus genes in some crops, such as soybean and cotton, has shown that these two proteins are directly involved in embryogenesis. They exhibit several isoforms that are essential for normal seed development.

The possible role of both genes in seed development is discussed in this review.
\end{abstract}

(c) 2009 Elsevier B.V. All rights reserved.

\section{Introduction}

The high nutritional value of seeds derives from the deposition of starch, lipids, proteins and essential minerals during their development. Alongside this process there is an accumulation of phytic acid and sugars which play a pivotal role in plants, acting as carbon currency and metabolic signals controlling many aspects of seed development, such as intracellular signaling regulation, signal transduction pathway, cell wall biogenesis, membrane formation and energy metabolism.

During the various stages of seed development, the availability of nutrients and the co-ordinated genetic program during embryo development are important in controlling the progression to seed completion.

In higher plants, embryogenesis plays a key role in establishing the correct pattern of cell division and orientation for embryo development and the accumulation of storage reserves. A better understanding of the molecular and physiological basis of this process is important in the search for ways of improving grain quality and yield.

Abbreviations: EMS, ethyl methanesulfonate; EST, express sequences tag; IAA, indole3-acetic acid; LPA, low phytic acid; MIPS, myo-inositol phosphate synthase; P-Sus, particulate Sus; SSH, suppressive subtractive hybridization; S-Sus, soluble Sus; Sus, sucrose synthase; T6P, trehalose-6-phosphate; TPP, trehalose-6-phosphate phosphatase; TPS, trehalose-6-phosphate synthase; UDP-glucose, uridine-5-diphosphoglucose.

* Corresponding author. Passage des Déportés, 2, 5030 Gembloux, Belgium. Tel.: +003281 622110; fax: +003281 614544 .

E-mail address: abid.g@fsagx.ac.be (G. Abid).
In flowering plants, early seed development requires a coordinated interaction between embryo and maternal tissues, particularly endosperm, a tissue that supports embryo development through nutrient metabolism and use.

Analysis of the early stages of development, however, is often difficult because the embryo is too small and is embedded inside the maternal tissue. Although many morphological and histological processes in embryogenesis are well known, the molecular and physiological events leading to seed formation are still far from being completely understood.

A great number of genes demonstrated by embryo mutant obtention might be needed for normal embryogenesis, and seem to be key regulators of the process (Tzafrir et al., 2004).

Several mutations in the endosperm, discovered in Arabidopsis thaliana (Kinoshita et al., 1999) and Ulmus minor (Lopez-Almansa et al., 2004), showed that embryo abortion was due, at least in part, to a defect in endosperm function supporting embryo growth and development.

Recently, studies of myo-inositol phosphate synthase (MIPS) in Arabidopsis (Mitsuhashi et al., 2008) and Glycine max (Chiera and Grabau, 2007), as well as mutated sucrose synthase (Sus) of Gossypium hirsutum using an RNAi approach (Ruan et al., 2008) revealed the critical role of both genes in early seed development through the regulation of endosperm formation. MIPS and Sus are both expressed in endosperm tissues in early seed development and during embryo formation. Chiera and Grabau (2007) reported that MIPS ensures the primary inositol supply for the early embryo, whereas Sus is involved in the cell wall biosynthesis of the endosperm. 
Interestingly, Lackey et al. (2003), using microscopic and biochemical analyses, detected MIPS expression in the cell wall and plasma membrane. Particulate Sus (P-Sus), the predominant form of Sus, has been shown to be localized in the plasma membrane and associated with the cellulose synthase for channeling UDP-glucose, the leading compound in cell wall biosynthesis during the developmental transition between primary and secondary wall synthesis. The relationship between MIPS and Sus in cell wall biosynthesis has not yet been reported.

In this review, we discuss the role of MIPS and Sus in normal embryogenesis and their possible functional role in plant biology, which includes cell wall biosynthesis, defense against biotic and abiotic stresses, and developmentally related processes.

\section{Diversity, gene structure and cellular localization of MIPS and Sus}

\subsection{Myo-inositol phosphate synthase (MIPS)}

The MIPS enzyme has been reported from a large number of living organisms, including higher plants and animals, parasites, fungi, green algae and bacteria. To date, more than 60 MIPS genes have been identified (Majumder et al., 2003). In higher organisms, the enzyme has two forms: cytosolic and organellar. Genes coding for cytosolic MIPS have been studied in detail and represents a multigene family in some plants. MIPS activity has been localized in cytosolic and plastidic compartments in pea (Pisum sativum) (Imhoff and Bourdu, 1973), Vigna radiata and Euglena gracilis (Adhikari et al., 1987), Oryza sativa (Hait et al., 2002; Ray Chaudhuri and Majunder, 1996), Citrus paradisi (Abu-abied and Holland, 1994), A. thaliana (Johnson and Sussex, 1995), Mesembryanthemum crystallinum (Ishitani et al., 1996), Zea mays and Hordeum vulgare (Keller et al., 1998), Brassica napus (Larson and Raboy, 1999), G. max (Iqbal et al., 2002), Sesamum indicum (Chun et al., 2003) and Phaseolus vulgaris (Johnson and Wang, 1996). Some experimental data have shown that chloroplastic MIPS activity increased when plants were exposed to strong light treatment and salinity stress (Ishitani et al., 1996).

The distribution and evolution of MIPS genes from a probable cenancestor to cyanobacteria, eubacteria and archaea, and ultimately to higher eukaryotes such plants and humans, indicate that MIPS started evolving from different sources (Majumder et al., 2003) and that MIPS enzyme activity is distributed in evolutionary diverse phyla, from eubacteria, archaebacteria, cyanobacteria, alguae, fungi to higher plants and animals.

The first MIPS reported from an archaebacterial source was from Archaeoglobus fulgidus (Chen et al., 2000). The characterization of Archaeoglobus MIPS showed that this enzyme was more active (by one order of magnitude) than other MIPS enzymes, and was functional at extremely high temperatures. Subsequently, MIPS proteins (with about 510 amino) acid were reported from other phylogenetically diverse organisms, such as red algae, bryophytes, gymnosperm and fungi. All the fungal MIPS proteins have an extended N-terminal end of about 28 amino acid residues, which is absent in the other MIPS Phylogenetic analysis of fungal MIPS has shown a high degree homology with the Saccharomyces MIPS gene.

Analyses of the total protein sequences (Majumder et al., 2003; Bachhawat and Mande, 2000) indicate that the eukaryotic MIPS family is homogenous but not similar to the prokaryotic MIPS genes. This could be explained by a monophyletic origin of the eukaryotic MIPS genes. In contrast, prokaryotic MIPS genes show a lower sequence similarity.

The genomic structure and organization of MIPS has been determined in several organisms, including yeast, A. thaliana, C. paradisi, Nicotiana tabacum, and $Z$. mays. All these sequences have shown regions of high conservation at the nucleotide level (Hegeman et al., 2001).

An alignment (Fig. 1) of the amino acid sequences of selected MIPS genes from eubacteria, archaea, parasites, fungi, plant and animal sources reveals several domains; the amino acid stretch GWGGNNG (domain 1), LWTANTERY (domain 2), NGSPQNTFVPGL (domain 3) and SYNHLGNNDG (domain 4), a stretch of eukaryotic MIPS identified as 'highly conserved' (Majumder et al., 2003). These four domains are involved in MIPS protein binding and are essential for MIPS functions, such as cofactor $\mathrm{NAD}^{+}$binding and reaction catalysis (Majumder et al., 1997).

Cytosolic MIPS was also isolated and characterized from Perilla frutescens and $A$. thaliana, indicating their presence during normal seed growth (Chung et al., 1999; Jin et al., 2000; Baud et al., 2004). In P. vulgaris, only one gene was identified, but Western blot analysis of the soluble proteins detected two distinct polypeptides during embryonic and postembryonic development. A small $33 \mathrm{kDa}$ protein was active during the globular stage of embryogenesis and in the mature roots. In contrast, another protein, with approximately $56 \mathrm{kDa}$, was present during the cotyledonary phase of embryogenesis and in the young roots (Johnson and Wang, 1996). Four genes were found in soybean (G. max), up-regulated in developing seeds and in flowers, leaves, roots and germinating cotyledons (Hegeman et al., 2001; Chappelle et al., 2006). In maize ( $Z$. mays), seven homologous MIPS sequences were mapped on different chromosomes (Larson and Raboy, 1999). In A. thaliana, two distinct MIPS genes were identified (Johnson and Sussex, 1995). In sesame (S. indicum), two or three sequences were isolated, and the expression pattern showed accumulation in leaves, stems, roots and developing seeds, but no expression in mature seeds (Chun et al., 2003).

\subsection{Sucrose synthase (Sus)}

Sucrose is the primary transport carbohydrate and the predominant form of translocated carbon in most higher plants. It is actively channeled into the different plant organs and is subsequently converted into sugars, such as hexose sugars, and into starch through a series of enzymatic reactions. These enzymes involved in sucrose metabolism play a pivotal role in plant growth and development (Koch, 2004). The primary enzymes in sucrose metabolism include sucrose phosphate synthase (EC 2.4.1.14), sucrose synthase (EC 2.4.1.13) and invertase (EC 3.2.1.26).

In the species examined to date, Sus is encoded by a small multigene family, Sus occurs as isoforms and is encoded by at least two genes. In many dicotyledonous species such as $A$. thaliana (Chopra et al., 1992), Solanum tuberosum (Fu and Park, 1995), Lycopersicon esculentum (Anguenot et al., 1999), Daucus carota (Sebkova et al., 1995), C. paradisi (Komatsu et al., 2002) and P. sativum (Barratt et al., 2001), two or more sucrose synthase genes have been found. In Vicia faba and G. max, however only one Sus has been identified (Arai et al., 1992).

A comparison of deduced amino acid sequences of plant Sus showed that this gene is expressed in the various taxonomic branches of land plants. However, dicotyledonous genes have clear similarities in sequence and overall structure, which distinguish them from monocotyledons and suggest a different evolutionary origin for both plant types. However, monocot/dicot orthologous groups (Fig. 2) supported by statistical analyses (bootstrap values) suggested conservation of Sus function in different species.

The prokaryotic Sus was isolated and biochemically characterized from cyanobacterium Anabaena sp. (Porchia et al., 1999). A comparison of deduced amino acid sequences originated from prokaryotic and plant Sus showed that a long divergent region extends to approximately 100 amino acids. These data suggest that the prokaryotic enzyme clearly diverges from the plant Sus reported to date (Curatti et al., 2000). Prokaryotic Sus is characterized by an $\mathrm{N}$-terminal amino acid stretch SELMQAILDS, whereas plant proteins are characterized by the following conserved region DTGGQVVY.

Sus can exist as homotetramers or heterotetramers. The molecular weight of each subunit is about $90 \mathrm{kDa}$, and the total native protein mass is $280-400 \mathrm{kDa}$ (Koch et al., 1992). Substrates for sucrose cleavage by Sus can be UDP, ADP, dTDP or GDP (Romer et al., 2004). 

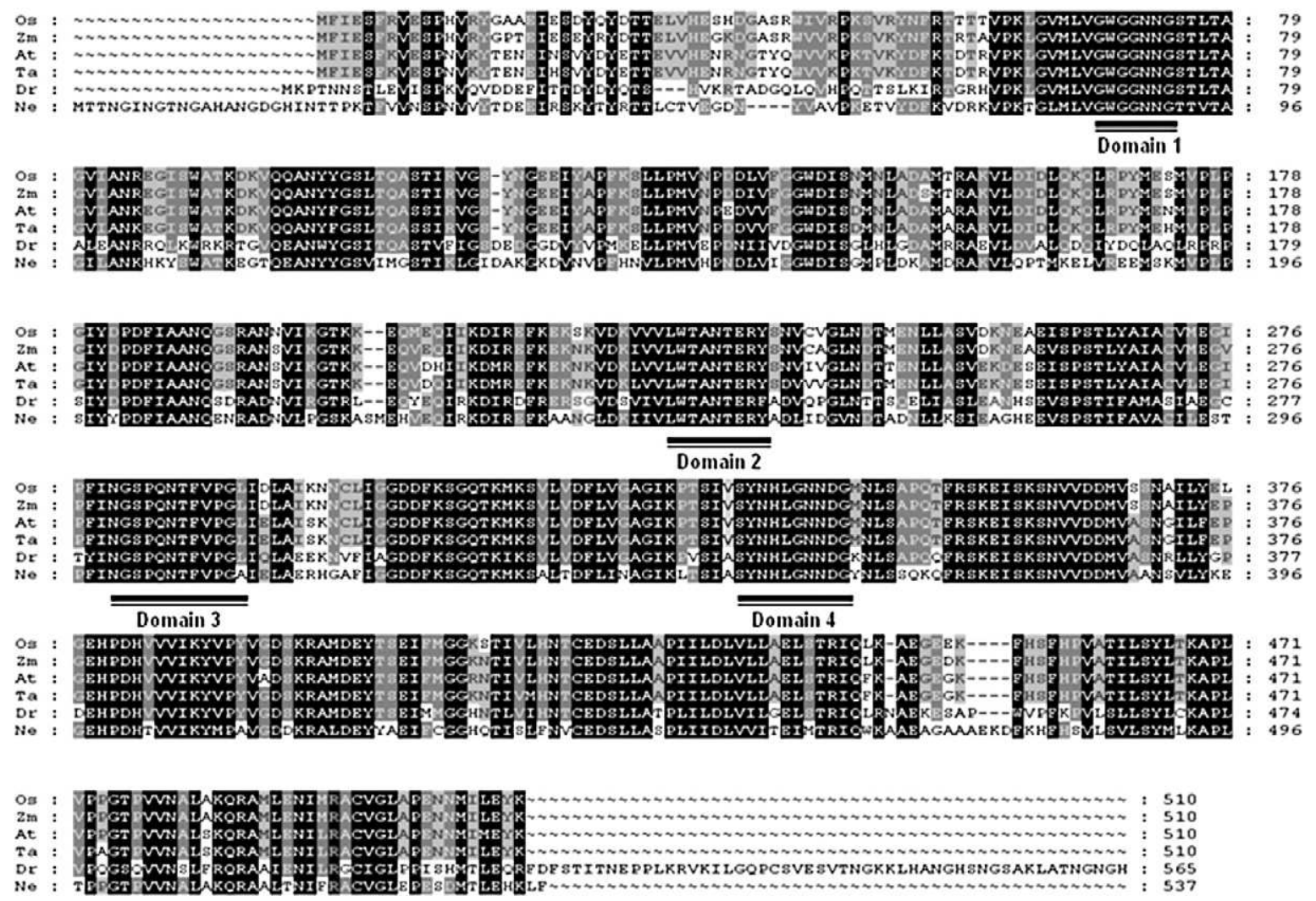

Fig. 1. Amino acid alignment of MIPS from different plant species; Oryza sativa (Os), Zea mays (Zm), Arabidopsis thaliana (At), Triticum aestivum (Ta), Drosophila (Dr) and Neurospora $(\mathrm{Ne})$. Dark and/or grey background appeared to highlight the positions. Where more than half of the accessions show identical and/or similar residues, respectively explain about domain 1 (GWGGNNG), domain 2 (LWTANTERY), domain 3 (NGSPQNTFVPGL), domain 4 (SYNHLGNNDG).

Kinetic measurements have shown that UDP-glucose is the most favorable substrate for Sus.

Five genes encoding Sus-like proteins have been discovered in the P. sativum genome (Barratt et al., 2001); they have a distinct expression pattern in plant organs and in organ development. In rice, six Sus genes have been characterized and the expression analysis suggests that Sus is involved in different growth processes (Hirose et al., 2008). Sus1 is expressed in elongating tissues, Sus3 and Sus4 are expressed mainly in caryopses, and Sus2 is expressed in all vegetative tissues. The roles of the recently isolated Sus5 and Sus6 genes are not clear yet.

Baud et al. (2004) showed that the A. thaliana genome contains six Sus genes; the isoforms they encode could be classified into three distinct groups based on their amino acid sequences and each isoform might have a specific function. Sus 5 and Sus6 are expressed in leaves, vascular cotyledons, petals, anthers and roots; Sus2 is expressed during seed development; SuS3 transcripts appear in mid seed development; SuS1 is widely expressed in vascular cotyledon, mature leaves and siliques; and the expression of SuS4 is clearly confined to stems, roots and germinating seeds (Bieniawska et al., 2007). In maize, three Sus genes have been identified (Carlson et al., 2002). Five Sus isoforms have been detected in Sorghum bicolor (Chourey et al., 1991). Silvente et al. (2003) indicated that there are at least two Sus genes in common beans ( $P$. vulgaris). One of these genes was expressed almost exclusively in nodules and the other was expressed in all the tissues tested, including cotyledons, stems, leaves, and roots. In sugarcane, two genes were identified and their level of expression varied significantly in different parts of the internodes and at the various development stages. These results suggested different levels of Sus activity (Schafer et al., 2004). In Pyrus pyrifolia fruit, two isoforms of Sus were purified. One was present mainly in young fruits, and the other was expressed particularly in mature fruit (Tanaze and
Yamaki, 2000). In V. faba, one enzyme was identified with a total molecular mass of $360 \mathrm{kDa}$ in cotyledons (Heather and Howard, 1992).

Sus is a phosphor protein. The role of the status in plants was to regulate the proteolytic turnover of the centrally important enzyme. Initial data indicated that a phosphorylated Sus protein was associated with the plasma membrane, whereas the dephosphorylated form was located in the cytosol (Carlson et al., 2002; Komina et al., 2002; Nakai et al., 1997). Nevertheless, Hardin et al. (2003) showed that Sus phosphorylation occurred on serine 15 (Ser15) and serine 170 (Ser170) and was associated with the 20S and 26S proteasomes.

In maize phosphorylation and dephosphorylation at Ser15 might be one of the regulatory mechanisms for modulating of Sus activity and/or subcellular localization.

To investigate the molecular evolution and phylogenetic relationships among Sus in plants, the entire SuS protein sequences were aligned by ClustalX and tree calculated by neighbor-joining algorithms. Using the full length of the alignment of all plant Sus selected, the distance method resulted in one tree. The Sus could be divided phylogenetically into six major groups; A, B, C, D, E, and F (as shown in Fig. 2); out the 52 plant genes, 17 were classified into group A. Each group could be further subdivided into subgroups of related proteins. The dramatic expansion of dicotyledonous and, to a lesser extent, monocotyledonous Sus genes, such as $Z$. mays and $O$. sativa in the D and $E$ group indicated that these two groups of genes should have expanded both before and after the monocot-dicot split. Interestingly, monocotyledonous Sus genes were clustered into one monocotyledon-specific group (C), suggesting a considerable divergence between monocotyledonous and dicotyledonous Sus. The recent expansion should be produced by polyploidy. In addition, the full-length Sus phylogeny showed that the evolution of dicotyledonous plant groups $A$ and $B$ was probably shaped by two duplication events: the first duplication produced group B and the common ancestor of group $A$; 


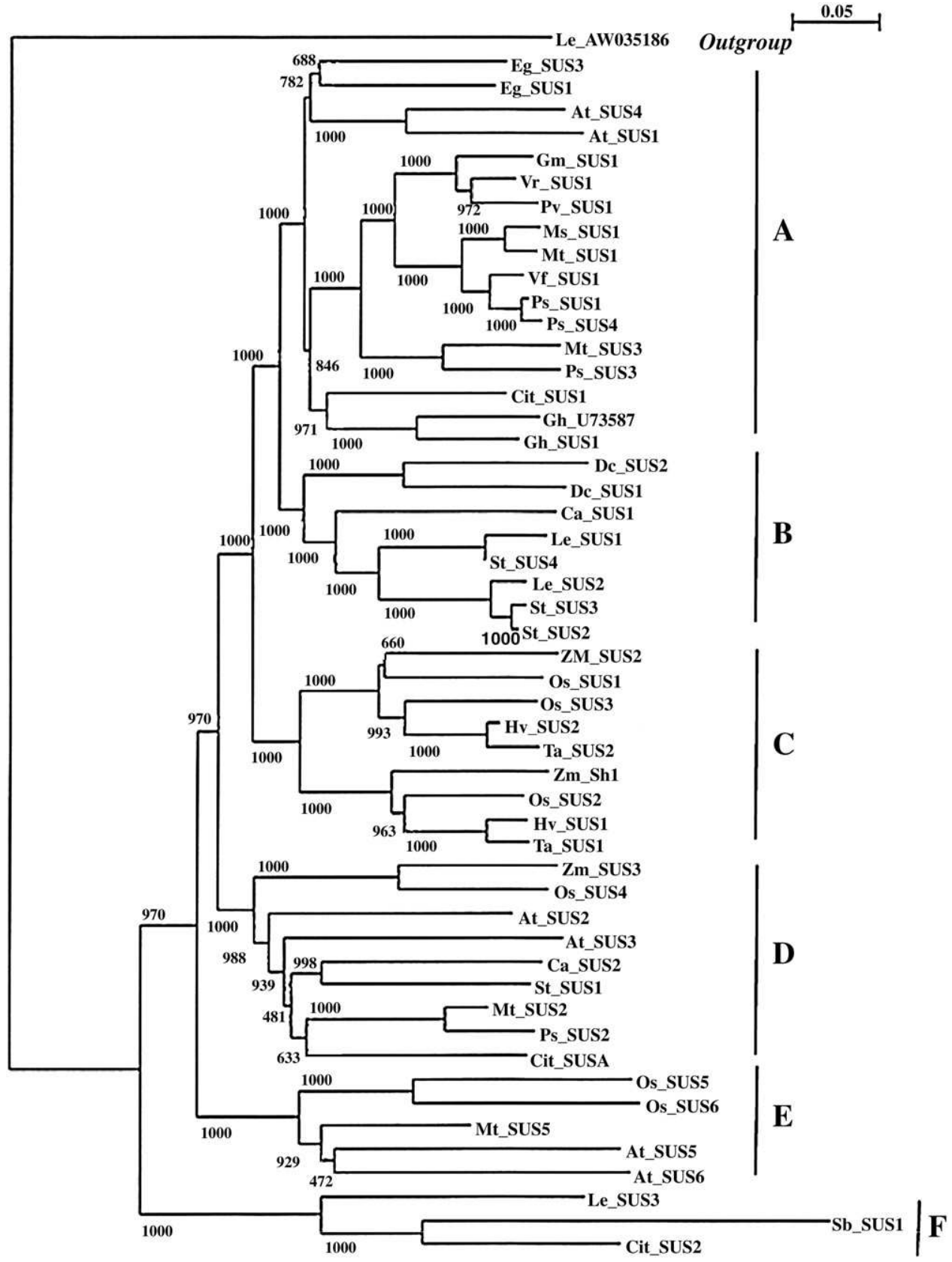

Fig. 2. Phylogenetic analysis of aligned amino acid sequences deduced from several cDNA Sus genes. The amino acid sequences of the ORFs were initially aligned using the program ClustalX-2.0.3-win. Distance analyses used the program Protdist and tree was calculated by neighbor-joing algorithms. To estimate phylogenetic relationships from the deduced amino acid sequences, a bootstrap majority-rule consensus tree was generated after 1000 cycles of bootstrap replicates using the parsimony method of the njplotWin95 software program. The numbers on the line denote frequency of occurrence of bootstrapping. The sequence sources were taken from the GenBank, TAIR, TIGR, EMBL-EBI and Gramene database: AY124703; Zea mays SUS1 (Zm_SUS1), L22296; Z. mays SUS2 (Zm_SUS2), X02400; Z. mays Sh1 (Zm_Sh1), At5g20830; Arabidopsis thaliana SUS1 (At_SUS1), At5g49190; A. thaliana SUS2 (At_SUS2), At4g02280; A. thaliana SUS3 (At_SUS3), At3g43190; A. thaliana SUS4 (At_SUS4), At5g37180; A. thaliana SUS5 (At_SUS5), At1g73370; A. thaliana SUS6 (At_SUS6), DQ227993; Eucalyptus grandis SUS1 (Eg_SUS1), DQ227994; E. grandis SUS3 (Eg_SUS3), AB022091; Citrus unshiu SUSA (Cit_SUSA), AB022092; C. unshiu SUS1 (Cit_SUS1), AB021745; C. unshiu SUS2 (Cit_SUS2), AJ012080; Pisum sativum SUS1 (Ps_SUS1), AJ001071; P. sativum SUS2 (Ps_SUS2), AJ311496; P. sativum SUS3 (Ps_SUS3), AF079851; P. sativium SUS4 (Ps_SUS4), Os03g0401300; Oryza sativa SUS1 (Os_SUS1), Os06g0194900; O. sativa SUS2 (Os_SUS2), Os07g0616800; O. sativa SUS3 (Os_SUS3), Os03g0340500; O. sativa SUS4 (Os_SUS4), Os04g0309600; O. sativa SUS5 (Os_SUS5), Os02g0831500; O. sativa SUS6 (Os_SUS6), X65871; Hordeum vulgare SUS1 (Hv_SUS1), Y15802; H. vulgare SUS2 (Hv_SUS2), AJ001117; Triticum aestivum SUS1 (Ta_SUS1), AJ000153; T. aestivum SUS2 (Ta_SUS2), X75332.1; Daucus carota SUS1 (Dc_SUS1), Y16091; D. carota SUS2 (Dc_SUS2), AY205302; Solanum tuberosum SUS1(St_SUS1), AY205084; S. tuberosum SUS2 (St_SUS2), U24088; S. tuberosum SUS3 (St_SUS3), AJ537575; S. tuberosum SUS4 (St_SUS4), U73588; Gossypium hirsutum SUS1 (Gh_SUS1), U73587; G. hirsutum U73587 (Gh_U73587), X69773; Vicia faba SUS1 (Vf_SUS1), TC67957; Medicago truncatula SUS1 (Mt SUS1), TC62069; M. truncatula SUS2 (Mt_SUS2), TC67958; M. truncatula SUS3 (Mt_SUS3), TC65865; M. truncatula SUS5 (Mt_SUS5), AF049487; Medicago sativa SUS1 (Ms_SUS1), AJ853494; Phaseolus vulgaris SUS1 (Pv_SUS1), AF030231; Glycine max SUS1 (Gm_SUS1), L19762; Lycopersion esculentum SUS1 (Le_SUS1), AJ011319; L. esculentum SUS2 (Le_SUS2), AF011534; L. esculentum SUS3 (Le_SUS3), AW035186; L. esculentum AW035186 (Le_AW035186), D10266; Vignia radiata SUS1 (Vr_SUS1), AM087674; Coffea arabica SUS1 (Ca_SUS1), AM087675; C. arabica SUS2 (Ca_SUS2), EF089747; Shorghum bicolor SUS1 (Sb_SUS1).

the last group would result from the second duplication. Interestingly, some putatively orthologous and paralogous pairs could be identified from the tree, such as Ms_SUS1/Mt_SUS1, Hv_SUS1/Ta_SUS1, Mt_SUS3/Ps_SUS3, Os_SUS5/Os_SUS6, etc. Group B is a far more homogenous group than any of the other groups. In summary, there are two or more monocotyledon and dicotyledon subgroups, a consequence of the large variation of divergence in these groups. A lower limit of divergence would result in group F. It is known that Sus 
genes were relatively well conserved over a long period of evolutionary time, and genes belonging to the same group might serve similar functions (Pramanik et al., 2005; Komatsu et al., 2002; Barratt et al., 2001; Sebkova et al., 1995). Therefore, the identification of orthologs and paralogs would greatly facilitate the functional annotation of uncharacterized Sus.

\section{Expression and regulation of MIPS and Sus genes}

\subsection{Relationship between MIPS and phytic acid biosynthesis}

In the higher organisms, as mentioned earlier, the MIPS enzyme has two forms, cytosolic and chloroplastic. The latter form, which is associated with thylakoid membranes, is regulated by light, whereas the former is involved in various important metabolic reactions (Majumder et al., 1997).

MIPS catalyses the conversion of glucose-6-phosphate (G6P) to myo-inositol 3-phosphate $(\operatorname{Ins}(3) \mathrm{P})$, the first product in the biosynthesis of inositol, phytic acid and other essential cellular components (Loewus and Murthy, 2000). Myo-inositol has a diverse biological role and participates in several cellular processes, including signal transduction, stress response, cell wall biogenesis, growth regulation, osmo-tolerance, IAA metabolism, membrane trafficking (Irvine and Schell, 2001) and in phytic acid synthesis.

Phytic acid is the predominant form in which phosphorus occurs in plants; it is a regulator of intercellular signaling and a phosphate storage form. This metabolite is accumulated mainly in seeds (up to $4-5 \%$ of dry weight) and pollen. Phytic acid, discovered in 1855 , has an inositol ring with six attached phosphate groups and is the most stable form of phosphorus (Oatway et al., 2001). Phytic acid is found in cereals and legumes grains, nuts, oilseeds, tubers, pollen, spores and organic soils (Oatway et al., 2001).
The biosynthetic pathway to phytic acid is summarized in Fig. 3. The first committed step in inositol synthesis involves the synthesis of myo-inositol-3-phosphate $(\operatorname{Ins}(3) \mathrm{P})$ from D-glucose-6-phosphate (G6P) catalysed by the MIPS enzyme. Ins(3)P is converted directly to phytic acid via sequential phosphorylation by two or more kinases.

The generated Ins(3)P is then dephosphorylated by inositol3-phosphatase to release free inositol. The inositol formed by this process from G6P can enter in different pathways and cellular compartments. There is evidence that the inositol backbone for phytic acid might be derived from MIPS activity. Inositol is crucial for normal plant growth and development. Plant cells metabolize myo-inositol via a number of pathways leading to phytate production.

\subsection{Sus key enzymes involved in sugar synthesis}

Sus is implicated in many aspects of plant metabolism. In various plants its activities play a major role in energy metabolism, controlling the mobilization of sucrose into various pathways that are important for the metabolic, structural and storage functions of the plant cell (Hess and Willmitzer, 1996).

A large part of the carbon produced during photosynthesis is channeled into synthesis of sugars, which play a key role as metabolic signals controlling many aspects of plant growth and development (Smeekens, 2000). Among the sugars, trehalose is a storage carbohydrate which protects the plant against stresses caused by various invertebrates and fungi (Goddijn and Van Dun, 1999).

The biosynthesis of trehalose (Fig. 4) involves the formation of trehalose-6-phosphate (T6P) from glucose-6-phosphate and UDPglucose from the enzyme trehalose-6-phosphate synthase (TPS). The latter is dephosphorylated to trehalose by trehalose-6-phosphate phosphatase (TPP) (Cabib and Lenoir, 1958). Higher plants have two ways of forming UDP-glucose, one based on Sus and the other on UDP-

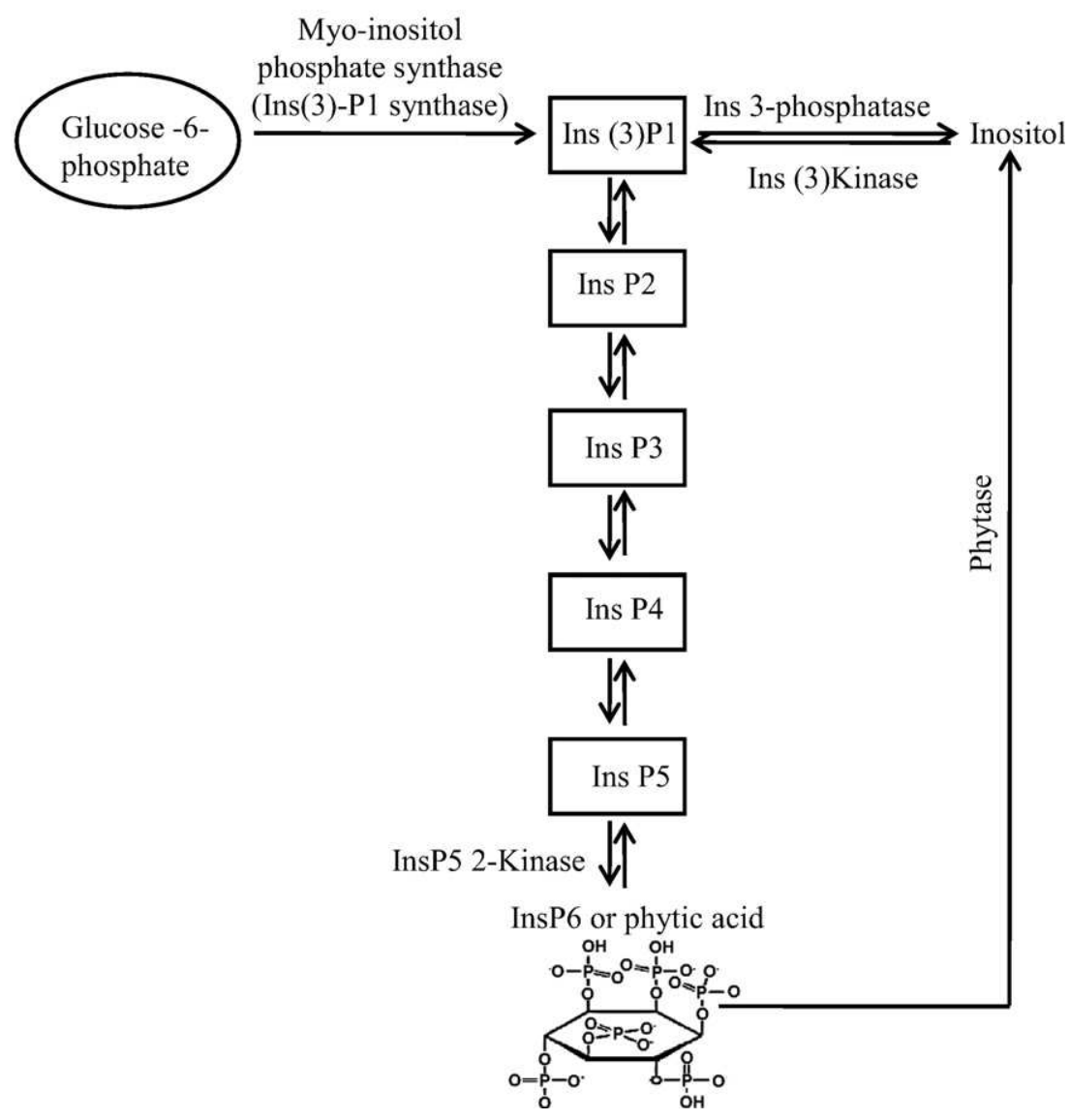

Fig. 3. Phytic acid metabolism in plants. Synthesis of phytic acid occurs from glucose-6-phosphate. 


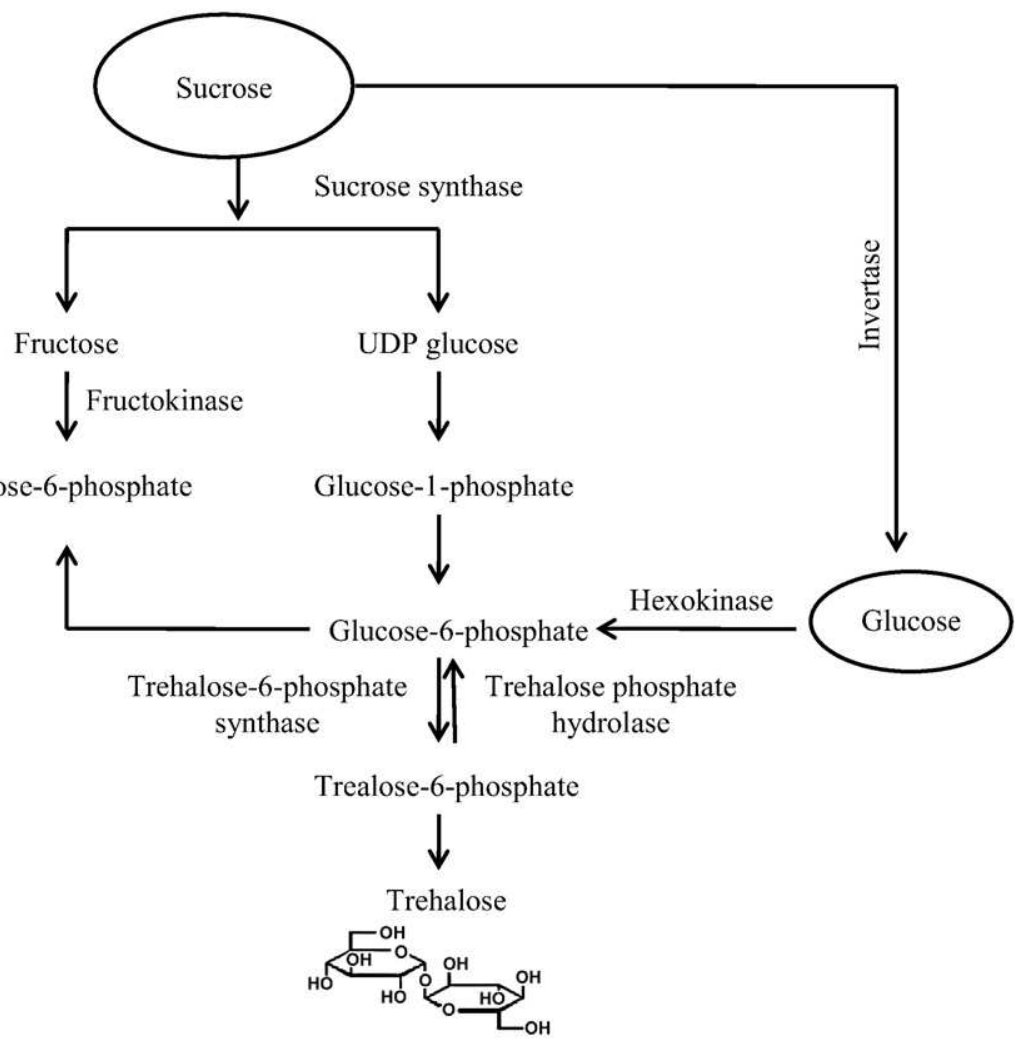

Fig. 4. Proposed degradation pathway for sucrose by sucrose synthase.

glucose pyrophosphorylase. T6P is of great importance in plant biology and has much potential in crop improvement (Eastmond and Graham, 2003). It is considered to be a central regulator of many cellular processes. Alternatively, trehalose metabolism could play a more direct role in regulating cell division as an integrator of nutritional status and growth (Gomez et al., 2006). It is thought to be responsible with invertase for sucrose mobilization in sink organs such as leaves, phloem, shoots, nodules, seed testas, embryos and fruits by converting sucrose and UDP into fructose and UDP-glucose (Craig et al., 1999).

Invertase is widely believed to be the main entry route of carbon as sucrose into the cellular metabolism in plants, and it is located mainly in the extracellular compartment (intercellular space and cell wall) and in the large central vacuole. This suggests an important physiological role of acid invertase as an entry point for carbohydrate into cellular metabolism, probably via control of sugar composition and metabolic fluxes, but also in sucrose portioning by regulating the cellular content of several major metabolites, including UDP-glucose, fructose, glucose, glucose-6-phosphate and fructose-6-phosphate. These metabolites regulate cell wall synthesis, the respiration rate, starch synthesis, storage in the vacuoles and other product synthesis. More interestingly, an optimal level of hexose sugars maintains cell division and expansion in the embryo (Borisjuk et al., 1998)

In general, Sus enzymes appear to be located primarily in sink tissues, where they facilitate sucrose import. Young leaves tend to have high Sus activity, but mature leaves do not (Hardin et al., 2003). In some plants, Sus activity is constant during leaf development (Baroja-Fernandez et al., 2001). In carrot (Dacus carota), Sus activity was highly correlated with both polypeptides and transcript levels, indicating that gene expression is regulated mainly at the mRNA level in the different tissues and organs of the developing carrot (Sebkova et al., 1995). In plants, the expression of Sus genes has been shown to be cell-specific, developmentally regulated or regulated by tissue carbohydrate status (Ruan et al., 1997). Sus is the major sucrose-cleaving enzyme involved in starch synthesis. According to Dejardin et al. (1997), Sus participates in the synthesis of sugars.

\section{Putative function of MIPS and Sus genes in seed plants}

\subsection{Myo-inositol phosphate synthase (MIPS)}

MIPS is a pivotal biosynthetic enzyme in the myo-inositol pathway, including the synthesis of phytic acid. It is found in uni and multicellular eukaryotes. A complete elimination of the myo-inositol pathway through MIPS gene mutation stops the formation of phytic acid, but it also disrupts the many other biochemical pathways needed for germination. Indeed, several mutant MIPS lines of maize (Raboy and Cook, 2000), barley (Larson et al., 1998; Ockenden et al., 2004), rice (Larson et al., 2000; Suzuki et al., 2007) and soybean (Wilcox et al., 2000; Hitz et al., 2002) show a reduction of phytic acid levels.

MIPS activity is halted by including deoxyglucose in growth media. Deoxyglucose is a substrate for MIPS and phosphorylated into the enzyme inhibitor deoxyglucose 6 -phosphate resulting in a reduction in the capacity of the cell to synthesize myo-inositol as well as in cell division (Biffen and Hanke, 1990). This confirms that MIPS signalizations are necessary for maintaining cell division.

Phytic acid, a natural plant antioxidant, is the main storage form of phosphorus in many plant tissues, especially bran and seed. More phosphorus as phytate is generally, not bioavailable to non-ruminant animals because they lack the digestive enzyme phytase, necessary to separate phosphorus from the phytate molecule. The bioavailability of phytate phosphorus can be increased by supplementing of the diet with phytase enzyme.

Viable low phytic acid mutant lines have been developed in several crop species in which the phytic acid levels drastically reduced in seeds. Low phytic acid (LPA) maize (Z. mays) mutant have been 
obtained by pollen treatment with EMS (ethyl methanesulfonate). A reduction in MIPS gene expression has been shown using an antisense approach (RNAi) in rice (O. sativa), barley (H. vulgare) and soybean (G. $\max$ ). These mutants have genetically reduced amounts of phytic acid (at least 70\%) in the seed. Raboy (2001) reported that a 90\% reduction in MIPS activity causes severe restriction of plant growth. Maize mutants with a phenotype of reduced phytic acid increased myoinositol and reduced amounts of myo-inositol phosphate intermediates in the seeds had a minimal effect on plant growth and development (Shi et al., 2005). These facts suggest that a high level of stored phytate is not necessary for seed viability and germination or seedling growth. Such mutants were isolated in soybean (Wilcox et al., 2000) and the genetic lesion was a single base change, which causes a replacement from lysine to asparagines in MIPS. The amino acid substitution reduces the specific activity of the seed expressed MIPS by about $90 \%$ (Hitz et al., 2002). Nunes et al. (2006), however, showed that total knockout of the soybean MIPS gene (GmMIPS1) leads to the abortion of soybean immature seeds. The characterization of mutant soybean indicates that MIPS genes play a critical role in the early developmental stages of the embryo. Using an immunological technique, the expression of the soybean MIPS was clearly detected in early developing seed, particularly in maternal tissues and in the developing embryo. At the globular embryo stage, MIPS is expressed in the outer integumentary layer (micropylar complex). Recently, in soybean, the MIPS enzyme showed association with calcium oxalate crystal idioblast (Chiera and Grabau, 2007), a complex that modulates physiological calcium levels in plant tissues and requires myo-inositol as a precursor to biosynthesis. In potato ( $S$. tuberosum), the suppression of MIPS activity using the RNAi approach showed a variety of morphological and physiological changes. The transgenic plants exhibited reduced apical dominance and altered leaf morphology, and provoked precocious leaf senescence and a decrease in overall tuber yield. These data indicate the essential role of myoinositol in plant physiology and development (Keller et al., 1998). Several low phytic acid (lpa) mutants have been generated; mapping some of them to early steps in inositol metabolism might explain the pleiotropic phenotypes. The alteration of the MIPS gene is a potential approach in the development of these low phytic acids plant. The characterization of these genotypes suggested that phytate and myoinositol, play a role as precursors to many compounds whose function in plants is linked to phosphorus storage, signal transduction, stress protection, hormonal homeostasis and cell wall biosynthesis (Hegeman et al., 2001). This MIPS role has a direct influence on plant growth and development. A complete elimination of the myo-inositol pathway would halt the formation of phytic acid, and it would disrupt many other biochemical pathways needed for germination, which in turn are fundamental in embryonic axis and cotyledon development (Stevenson et al., 2000).

MIPS activity has been localized in different organs (roots, leaves, suspensors and/or embryos, and cotyledons), and different forms of MIPS at different stages of development reflect regulatory controls at the transcriptional and translational levels (Adhikari et al., 1987). MIPS is also expressed during embryonic and postembryonic development in P. vulgaris (Johnson and Wang, 1996). These data indicate the important role of MIPS not only in basic metabolism, but also in the establishment of developmental programs. Over-expression in transgenic A. thaliana elevated the content of inositol and MIPS activity, but it did not affect plant growth or salt tolerance (Smart and Flores, 1997). The localization of three isoforms of the MIPS gene was investigated by Mitsuhashi et al. (2008) in developing Arabidopsis seeds. Immunolocalization, immunofluorescent microscopy and Western blot analysis indicated that AtMIPS proteins appear to be specifically located in the cytosol of the endosperm, but not in the embryo. Phytic acid is therefore accumulated during seed development. These data suggested, however a possible interaction between endosperm and embryo to ensure phytic acid synthesis.

\subsection{Sucrose synthase (Sus)}

Sus is important for the carbon metabolism used by storage tissues in many species and is located mainly in transport and sink tissues (Koch et al., 1996). It occupies a critical position in the cleavage of sucrose imported into developing plant structures, but members of Sus gene families can have diverse functions. Its substrate (sucrose) and product (UDP-glucose) are involved in carbon partitioning and signal sensing. For this reason, considerable effort has been made to study the biochemical properties of Sus, its localization, gene expression and regulation.

Sus provides the energy for sucrose channeling from the apoplast, in starch storage and in callose biosynthesis.

The level of soluble sugars, such as glucose and sucrose, is known to regulate developmental processes from embryo development to senescence. In embryo, storage reserve protein gene expression and accumulation such as $2 \mathrm{~S}$ albumin and $12 \mathrm{~S}$ globulin in Arabidopsis (Baud et al., 2002), are differentially induced at the transition from torpedo to early cotyledon phase. Interestingly, storage reserve gene induction and reserve accumulation have been reported occurring in embryo lethal mutants where growth stops as early as the globular stage of embryo development. Craig et al. (1999) studied Sus activities in pea rug4 mutants. They showed that mutations at the rug4 locus caused a considerable and specific reduction in Sus activity in the developing embryo, leading to a reduction in starch contents and a smaller reduction in Sus activity in testas and leaves. In transgenic potato (S. tuberosum) plants obtained with reduced Sus activity had a lower starch content than the control plants (Zrenner et al., 1995). In mutants of cotton (Gossypium hirstum), the suppression of Sus activity in only the maternal seed represses fiber development without affecting embryo development and seed size. Additional suppression in the endosperm and embryo inhibits their development, which blocks the formation of adjacent seed coat transfer cells and halts seed development entirely (Ruan et al., 2003). Recently cotton mutants have been obtained by silencing the expression of Sus in the endosperm using the RNAi approach, halting early seed development. The results provide new insight into the roles of Sus in controlling plant cell and early seed development through the regulation of endosperm formation (Ruan et al., 2008).

The expression of Sus has been manipulated by the antisense technique in transgenic carrot ( $D$. carota). The results of phenotypical and biochemical examination of the transgenic plants demonstrated that Sus in carrots is a major determinant of plant growth (Tang and Sturm, 1999). In carrots, the two Sus isoforms have different expression patterns; one is expressed exclusively in flowers, and the other in stems, roots, flowers, and maturing seed (Sturm et al., 1999). The phenotype of the antisense plants clearly differed from that of the control plants. The leaves and roots were markedly smaller, and the antisense plantlets with the lowest Sus activity also developed into smallest plants.

In maize, three Sus genes have been identified: SH1, SuS1 and SuS3 (Carlson et al., 2002). The loss of their functions suggested their involvement in starch biosynthesis and cell wall formation during endosperm formation (Chourey et al., 1998).

The A. thaliana genome contains six Sus genes. An examination of knockout mutants of all the isoforms caused, in particular, a loss of sugar, starch, cellulose content and weight and lipid content in mature seeds (Martin et al., 1993).

In contrast, however a loss of both isoforms (SuS2 and SuS3) most highly expressed in developing Arabidopsis seeds had no effect on final seed weight and lipid content (Bieniawska et al., 2007). The expression of different isoforms Sus in Arabidopsis did not show strong tissue-specific expression. Fallahi et al. (2008) reported that Arabidopsis Sus were expressed in the same stages of seed development and in several tissues from plants including immature and mature rosettes, cauline leaves, stems, flowers, and developing 
siliques. These data illustrated the potential role of Sus enzymes in carbon partitioning during the different stages of seed and plant development.

When plant growth occurs under controlled light conditions, the lack of any obvious phenotypes among the single and double knockout mutants showed a specific requirement for Sus activity in maize, pea, cotton and potato. In cotton and carrot species, mutant and antisense plants deficient in specific isoforms of SuS had marked phenotypes (Tang and Sturm, 1999; Ruan et al., 2003). Pea mutants (rug4) lacking an isoform very similar to Sus1 and Sus4 of A. thaliana were characterized by reduced seed mass and starch content, and they failed to assimilate $\mathrm{N}_{2}$ in their Rhizobium root nodules (Craig et al., 1999).

It is known that sugar metabolism is affected by temperature stress. In cabbage (Brassica oleracea) seedlings, the starch, hexose and sucrose content and the Sus activities increased during cold treatment. These data suggest that Sus is regulated by cold acclimation and play an important role in sugar accumulation and the acquisition of freezing tolerance in the leaves of cabbage seedlings (Sasaki et al. 2001). The Sus gene, however, is also present in other tissues such as roots and shoots, and its expression is involved in the response to biotic and abiotic stress (Zeng et al., 1999).

\subsubsection{Putative function of Sus in cell wall biosynthesis}

Sus plays a major role in the degradation of sucrose (sucrose + $\mathrm{UDP} \leftrightarrow \mathrm{UDP}$ - glucose + fructose). All these substrates constitute a start compound for cellulose biosynthesis. Cellulose is a compound with a critical role as the foundational polymer in plant cell walls. Studies of transformed carrot (Sturm et al., 1999) and maize (Chen and Chourey, 1989; Carlson and Chourey, 1996) plants showed Sus expression changes and cell wall synthesis disruption. These data strongly suggested a possible role of Sus in the biosynthesis of primary and secondary wall cellulose synthesis. During the formation of the primary cell wall, cellulose is not abundant. Walls contain $20-25 \%$ cellulose and non-cellulosic polymers such as xyloglucan and galacturonic acid (Meinert and Delmer, 1977). Towards the end of the development of the primary cell wall, secondary cell wall deposition begins via enhanced cellulose synthesis. In cotton (G. hirsutum and Gossypium barbadense), the rate of cellulose synthesis increases more than a hundredfold during the formation of the secondary cell wall (Basra and Malik, 1984). At this stage, about $80 \%$ of the imported carbon is channeled to cellulose. Amor et al. (1995) reported the presence of two forms of Sus: particulate Sus (P-Sus) associated with the plasma membrane, and soluble Sus (S-Sus). Using cryogenic electron microscopic methods and immunofluorescence, Amor et al. (1995) studied both forms of Sus activity during cotton fiber synthesis; the data showed that P-Sus channels UDPglucose to cellulose synthesis in the plasma membrane. Interestingly, Pear et al. (1996) identified three genes involved in cellulose synthesis in cotton; one was up-expressed during primary cell wall synthesis, and the other genes were highly expressed in secondary cell wall synthesis. Subsequently, 10 genes in Arabidopsis (Turner and Somerville, 1997), eight in maize and only one in rice (Holland et al., 2000) were identified. The discovery and cloning of the gene that encodes cellulose synthase was carried out after searching for plant orthologs in the corresponding bacterial cellulose synthase gene from Actobacter xilinum and Agrobacterium tumefaciens. The characterization of the cellulose synthase complexes in the plasma membrane appears to play a key role in the shape of the cellulose molecules synthesized. Both forms of Sus (P-Sus and S-Sus) adjust intracellular carbon partitioning, although, P-Sus could be critical for partitioning carbon to other different compounds such as cellulose. In contrast S-Sus could partition carbon in response to other demands, such as respiration and deposition of storage. The predominant form of Sus is P-Sus, which channels of UDP-glucose directly into cellulose synthase for cellulose cell wall biosynthesis.

Sucrose synthesis and degradation with Sus can occur intensively during high-rate cellulose synthesis for secondary wall deposition and at a lower level during primary wall synthesis. It is clear however, that Sus, particularly the P-Sus form, plays an important role in primary and secondary wall cellulose biosynthesis in plants.

\section{Conclusions}

Essential genes are required for growth and seed development (Harada, 1999). MIPS, the pivotal biosynthetic enzyme in inositol metabolism, is the only enzyme known to catalyse the conversion of glucose 6-phosphate to inositol phosphate, a precursor for many compounds whose functions in plants are involved in phosphorus storage, signal transduction, stress protection, hormonal homeostasis and cell wall biosynthesis.

Sus occupies a central position in the cleavage of sucrose, which in turn might regulate the rate of carbon imported into developing seeds, including the supply of carbon to the developing embryo, and control gene expression in it. However, SuS is believed to play an important role in cell growth by providing UDP-glucose and fructose through sucrose cleavage for energy metabolism and cell wall biosynthesis. Some members of the MIPS and Sus gene families could have diverse functions.

MIPS and Sus genes are essential for the higher plants. They are considered to be central regulators of several metabolisms and physiological processes in plant growth and seed development.

\section{References}

Abu-abied, M., Holland, D., 1994. The gene cINO1 from Citrus paradise is highly homologous to tur1 and Ino1 from the yeast and Spirodela encoding for myoinositol 1-phosphate synthase. Plant Physiol. 106, 1689.

Adhikari, J., Majumder, A.L., Bhaduri, T.J., Dasgupta, S., 1987. Chloroplast as a local of L-myo-inositol-1-phosphate synthase. Plant Physiol. 85, 611-614.

Amor, Y., Haigler, C.H., Wainscott, M., Johnson, S., Delmer, D.P., 1995. A membraneassociated form of sucrose synthase and its potential role synthesis of cellulose and callose in plants. Proc. Natl. Acad. Sci. U. S. A. 92, 9353-9357.

Anguenot, R, Yelle, S, Nguyen-Quoc, B, 1999. Purification of tomato sucrose synthase phosphorylated isoforms by Fe (III)-immobilized metal affinity chromatography. Acta Biochem. Biophys. 365, 163-169.

Arai, M., Mori, H., Imaseki, H., 1992. Expression of the gene for sucrose synthase during growth of mung bean seedlings. Plant Cell Physiol. 33, 503-506.

Bachhawat, N., Mande, S.C., 2000. Complex evolution of the inositol-1-phosphate synthase gene among archaea and Eubacteria. Trends Genet. 16, 111-113.

Baroja-Fernandez, E., Munoz, F.J., Akazawat, T., Pozueta-Romero, J., 2001. Reappraisal of the currently prevailing model of starch biosynthesis in photosynthetic tissues: a proposal involving the cytosolic production of ADP-glucose by sucrose synthase and occurrence of cyclic turnover of starch in the chloroplast. Plant Cell Physiol. 42 1311-1320.

Barratt, D.H.P., Barber, L., Kruger, N.J., Smith, A.M., Wang, T.L., Martin, C., 2001. Multiple, distinct isoforms of sucrose synthase in pea. Plant Physiol. 127, 655-664.

Basra, A.S., Malik, C.P., 1984. Development of the cotton fiber. Inter. Rev. Cytol. 89, 65-113.

Baud, S., Boutin, J.P., Miquel, M., Lepiniec, L., Rochat, C., 2002. An integrated overview of seed development in Arabidopsis thaliana ecotype WS. Plant Physiol. Biochem. 40, 151-160.

Baud, S., Vaultier, M.N., Rochat, C., 2004. Structure and expression profile of the sucrose synthase multigene family in Arabidopsis. J. Exp. Bot. 55, 397-409.

Bieniawska, Z., et al., 2007. Analysis of the sucrose synthase gene family in Arabidopsis. Plant J. 49, 810-828.

Biffen, M., Hanke, D.E., 1990. Reduction in the level of intacellular myo-inositol in cultured soybean (Glycine max L.) cells inhibits cell division. Biochem. J. 265, 809-814.

Borisjuk, L., Wang, T.L., Rolletscheck, H., Wobus, U., Weber, H., 1998. High-resolution histographical mapping of glucose concentrations in developing cotyledons of Vicia faba in relation to mitotic activity and storage processes: glucose as a possible developmental trigger. Plant J. 15, 583-591.

Cabib, E., Lenoir, L.F., 1958. The biosynthesis of trehalose-6-phosphate. J. Biol. Chem. 231, 259-275.

Carlson, S.J., Chourey, P.S., 1996. Evidence for plasma membrane-associated forms of sucrose synthase in maize. Mol. Gene Genet. 252, 303-310.

Carlson, S.J., Chourey, P.S., Helentjaris, T., Datta, R., 2002. Gene expression studies on developing Kernels of maize synthase (SuSy) mutants show evidence for a third SuSy gene. Plant Mol. Biol. 49, 15-29.

Chappelle, A.S., Scaboo, A.M., Wu, X., Nguyen, H., Pantalone, V.R., Bilyeu, K.D., 2006 Characterization of the MIPS gene family in Glycine max L. Plant Breed. 125, 493-500.

Chen, Y.C., Chourey, P.S., 1989. Spatial and temporal expression of the two sucrose synthase genes in maize: immunohistological evidence. Theor. Appl. Genet. 78, 553-559. 
Chen, L., Zhou, C., Yang, H., Roberts, M.F., 2000. Inositol 1-phosphate synthase from Archaeoglobus fulgidus is a class II aldolase. Biochemistry 39, 12415-12423.

Chiera, J.M., Grabau, E.A., 2007. Localization of myo-inositol phosphate synthase (GmMIPS-1) during the early stages of soybean seed development. J. Exp. Bot. 58, 2261-2268.

Chopra, S., Del-Favero, J., Dolferus, K., Jacobs, M., 1992. Sucrose synthase of Arabidopsis: genomic cloning and sequence characterization. Plant Mol. Biol. 18, 131-134.

Chourey, P.S., Taliercio, E.W., Carlson, S.J., Ruan, Y.L., 1998. Genetic evidence that the tow osozymes of sucrose synthase present in developing maize endosperm are critical, one for cell wall integrity and the other for starch biosynthesis. Mol. Gene Genet. 259, 88-96.

Chourey, P.S., Taliercio, E.W., Kane, E.J., 1991. Tissue specific expression and anaerobically induced posttranscriptional modulation of sucrose synthase genes in Sorghum bicolor M. Plant Physiol. 96, 485-490.

Chun, J.A., et al., 2003. Isolation and characterization of a myo-inositol 1-phosphate synthase cDNA from developing sesame (Sesamum indicum L.) seeds: functional and differential expression, and salt-induced transcription during germination. Planta 216, 874-880.

Chung, C.H., Kim, J.L., Lee, Y.C., Choi, Y.L., 1999. Cloning and characterization of a seedspecific fatty acid desaturase cDNA from Perilla frutescens. Plant Cell Physiol. 40, 114-118.

Craig, J., et al., 1999. Mutation at the rug4 locus alter the carbon and nitrogen metabolism of pea plants through an effect on sucrose synthase. Plant J. 17, 353-362.

Curatti, L., Porchia, A.C., Herrera-Estrella, L., Salerno, G.L., 2000. A prokaryotic sucrose synthase gene (sus $A$ ) isolated from a filamentous nitrogen-fixing cyanobacterium encodes a protein similar to those of plants. Planta 211, 729-735.

Dejardin, A., Rochat, C., Mougenest, S., Boutin, J.P., 1997. Purification, characterization and physiological role of sucrose synthase in the pea seed coat (Pisum sativum L.). Planta 201, 128-137.

Eastmond, P.J., Graham, I.A., 2003. Trehalose metabolism: a regulatory role for trehalose-6-phosphate? Curr. Opin. Plant Biol. 6, 231-235.

Fallahi, H., Scofield, G.N., Badger, M.R., Chow, W.S., Furbank, R.T., Ruan, Y.L., 2008. Localisation of sucrose synthase in developing seed and siliques of Arabidopsis thaliana reveals diverse roles for SUS during development. J. Exp. Bot. 59, 3283-3295.

Fu, H., Park, W.D., 1995. Sink and vascular-associated sucrose synthase functions are encoded by different gene classes in potato. Plant Cell 7, 1369-1385.

Goddijn, O.J.M., Van Dun, K., 1999. Trehalose metabolism in plants. Trends Plant Sci. 4, 315-319.

Gomez, L.D., Baud, S., Gilday, A., Li, Y., Graham, I.A., 2006. Delayed embryo development in the Arabidopsis trehalose-6-phosphate synthase 1 mutant is associated with altered cell wall structure, decreased cell division and starch accumulation. Plant J. 46, 69-84.

Hait, N.C., Ray Chaudhuri, A., Das, A., Bhattacharyya, S., Majunder, A.L., 2002. Processing and activation of chloroplast L-myo-inositol 1-phosphate synthase from Oryza sativa requires signals from both light and salt. Plant Sci. 162, 559-568.

Harada, J.J., 1999. Signaling in plant embryogenesis. Curr. Opin. Plant Biol. 2, 23-27.

Hardin, S.C., Tang, G.O. Scholz, A., Holtgraewe, D., Winter, H., Huber, S.C., 2003. Phosphorylation of sucrose synthase at serine 170: occurrence and possible role as a signal for proteolysis. Plant J. 35, 588-603.

Heather, A.R., Howard, V.D., 1992. Purification and characterization of sucrose synthase from the cotyledons of Vicia faba L. Plant Physiol. 100, 1008-1013.

Hegeman, C.E., Good, L.L., Grabau, E.A., 2001. Expression of D-myo-inositol-3-phosphate synthase in soybean. Implication for Phytic acid biosynthesis. Plant Physiol. 125, $1941-1948$.

Hess, H., Willmitzer, L., 1996. Expression analysis of a sucrose synthase gene from sugar beet (Beta vulgaris L.). Plant Mol. Biol. 30, 863-872.

Hirose, T., Scofield, G.N., Terao, T., 2008. An expression analysis profile for the entire sucrose synthase gene family in rice. Plant Sci. 174, 534-543.

Hitz, W.D., Carlson, T.J., Kerr, P.S., Sebastien, S.A., 2002. Biochemical and molecular characterization of a mutation that confers a decreased raffinosaccharide and phytic acid phenotype on soybean seeds. Plant Physiol. 128, 650-660.

Holland, N., Holland, D., Helentjaris, T., Dhugga, K.S., Xoconostle-Cazares, B., Delmer, D.P., 2000. A comparative analysis of the plant cellulose synthase (CesA) gene family. Plant Physiol. 123, 1313-1324.

Imhoff, V., Bourdu, R., 1973. Formation d'inositol par les chloroplasts isoles de pois. Phytochemistry 12, 331-336.

Iqbal, M.J., et al., 2002. A pyramid of loci for partial resistance to Fusarium salani f. sp. Glysines maintains Myo-inositol-1-phosphate synthase expression in soybean roots. Theor. Appl. Genet. 105, 1115-1123.

Irvine, R.F., Schell, M.J., 2001. Back in the water: the return of the inositol phosphates. Nat. Rev. Mol. Cell Biol. 2, 327-338.

Ishitani, M., Majumder, A.L., Borhouser, A., Michalowski, C.B., Jensen, R.G., Bohnert, H.J., 1996. Coordinate transcriptional induction of myo-inositol metabolism during environmental stress. Plant J. 9, 537-548.

Jin, U.H., et al., 2000. Characterization of a methionine-rich storage protein cDNA from perilla (Perilla frutescens) seed. Plant Physiol. 27, 701-707.

Johnson, M.D., Sussex, I.M., 1995. 1-L-myo-inositol 1-phosphate synthase from Arabidopsis thaliana. Plant Physiol. 107, 613-619.

Johnson, M.D., Wang, X., 1996. Differentially expressed forms of 1-L-myo-inositolphosphate synthase (EC5.5.1.4) in Phaseolus vulgaris. J. Biol. Chem. 271, 17215-17218.

Keller, R., Brearley, C.A., Trethewey, R.N., 1998. Reduced inositol content and altered morphology in transgenic potato plants inhibited for 1D-myo-inositol 3-phosphate synthase. Plant J. 16, 403-410.

Kinoshita, T., Yadegari, R., Harada, J.J., Goldberg, R.B., Fischer, R.L., 1999. Imprinting of the MEDA polycomb gene in the Arabidopsis endosperm. Plant Cell 11, 1945-1952.
Koch, K.E., 2004. Sucrose metabolism regulatory mechanisms and pivotal roles in sugar sensing and plant development. Curr. Opin. Plant Biol. 7, 235-246.

Koch, K.E., Nolte, K.D., Duke, E.R., McCarty, D.R., Avigne, W.T., 1992. Sugar levels modulate differential expression of maize sucrose synthase genes. Plant Cell 4, $59-69$.

Koch, K.E., Wu, Y., Xu, J., 1996. Sugar and metabolic regulation of genes for sucrose metabolism: potential influence of maize sucrose synthase and soluble invertase response on carbon partitioning and sugar sensing. J. Exp. Bot. 47, 1179-1185.

Komatsu, A., Moriguchi, T., Koyama, K., Omura, M., Akihama, T., 2002. Analysis of sucrose synthase genes in citrus suggests roles and phylogenetic relationships. J. Exp. Bot. 53, 61-71.

Komina, O., Zhoo, Y., Sarath, G., Chollet, R., 2002. In vitro phosphorylation of membrane and soluble forms of soybean nodule sucrose synthase. Plant Physiol. 129, 1664-1673.

Lackey, K.H., Pope, P.M., Johonson, M.D., 2003. Expression of 1L-myoinositol-1-phosphate synthase in organelles. Plant Physiol. 132, 2240-2247.

Larson, S.R., Raboy, V., 1999. Linkage mapping of maize and barley myo-inositol 1-phosphate synthase DNA sequences: correspondence with low phytic acid mutation. Theor. Appl. Genet. 99, 27-36.

Larson, S.R., Young, K.E., Cook, A., Balke, T.K., Raboy, V., 1998. Linkage mapping tow mutations that reduce phytic acid content of barley grain. Theor. Appl. Genet. 97, $141-146$.

Larson, S.R., Rutger, J.N., Young, K.A., Raboy, V., 2000. Isolation and genetic mapping of a non lethal rice (Oryza sativa L) low phytic acid 1 mutation. Crop Sci. 40,1397-1405.

Loewus, F.A., Murthy, P.P.N., 2000. Myo-Inositol metabolism in plant. Plant Sci. 150, $1-19$.

Lopez-Almansa, J.C., Yeung, E.C., Gil, L., 2004. Abortive seed development in Ulmus minor (Ulmaceae). Bot. J. Linn. Soc. 145, 455-465.

Majumder, A.L., Johnson, M.D., Henry, S.A., 1997.1L-myo-inositol 1-phosphate synthase. Acta Biochim. Biophys. 1348, 245-256.

Majumder, A.L., Chatterjee, A., Ghosh, D.K., Majee, M., 2003. Diversification and evolution of L-myo-inositol 1-phosphate synthase. FEBS Lett. 553, 3-10.

Martin, J., Frommer, W.B., Salanoubat, M., Willmitzer, L., 1993. Expression of an Arabidopsis sucrose synthase gene indicates a role in metabolization of sucrose both during phloem loading and in sink organs. Plant J. 4, 367-377.

Meinert, M.C., Delmer, D.P., 1977. Changes in biochemical composition of the cell wall of the cotton fiber during development. Plant Physiol. 59, 1088-1097.

Mitsuhashi, N., et al., 2008. Localization of myo-inositol-1-phosphate synthase to the endosperm in developing seeds of Arabidopsis. J. Exp. Bot. 59, 3069-3076.

Nakai, T., Tonouchi, N., Tsuchida, T., Mori, H., Sakai, F., Hayashi, T., 1997. Synthesis of asymmetrically labeled sucrose by a recombinant sucrose synthase. Biosci. Biotechnol. Biochem. 61, 1955-1956.

Nunes, A.C.S., Vianna, G.R., Cuneo, F., Amaya-Farfan, J., Capdeville, G., Rech, E.L., 2006 RNAi-mediated silencing of the myo-inositol-1-Phosphate synthase gene (GmMIPS1) in transgenic soybean inhibited seed development and reduced phytate content. Planta 224, 125-132.

Oatway, L., Vasathan, T., Helm, J.H., 2001. Phytic acid. Food Rev. Int. 17, 419-431.

Ockenden, I., et al., 2004. Characterization of the storage of phosphorus, inositol phosphate and cations in grain tissues of four barley (Hordeum vulgare L.) low phytic acid genotypes. Plant Sci. 167, 1131-1142.

Pear, J., Kawagoe, Y., Schreckengost, W., Delmer, D.P., Stalker, D., 1996. Higher plants contain homologs of the CelA genes that encode the catalytic subunit of the bacterial cellulose synthases. Proc. Natl. Acad. Sci. U. S. A. 93, 12642-12673.

Porchia, A.C., Curatti, L., Salerno, G.L., 1999. Sucrose metabolism in cyanobacteria: sucrose synthase from Anabaena sp. strain PCC 7119 is remarkably different from the plant enzyme with respect to substrate affinity and amino-terminal sequence. Planta 210, 34-40.

Pramanik, B.K., Matsui, T., Suzuki, H., Kosugi, Y., 2005. A sucrose synthase from broccoli: cDNA cloning sequencing and its expression during storage. Biotechnology 4 288-295.

Raboy, V., 2001. Seeds for a better future "low phytate" grains help to overcome malnutrition and reduce pollution. Trends Plant Sci. 6, 458-462.

Raboy, V., Cook, A., 2000. Origin and seed phenotype of maize low phytic acid 1-1 and low phytic acid 2-1. Plant Physiol. 124, 355-368.

Ray Chaudhuri, A., Majunder, A.L., 1996. Salinity induced enhancement of L-myo-inositol 1phosphate synthase in rice (Oryza sativa L.). Plant Cell Environ. 19, 1437-1442.

Romer, U., Schrader, H., Gunther, N., Netelstroth, N., Frommer, W., Elling, L., 2004. Expression purification and characterization of recombinant sucrose synthase 1 from Solanum tuberusum L. For carbohydrate engineering. J. Biotechnol. 107, 135-149.

Ruan, Y.L., Chourey, P.S., Delmer, D.P., Perez-Grau, L., 1997. The differential expression of sucrose synthase in relation to diverse patterns of carbon partitioning in developing cotton seed. Plant Physiol. 115, 375-385.

Ruan, Y.L., Llewellyn, D.J., Furbank, R.T., 2003. Suppression of sucrose synthase gene expression represses cotton fibre cell initiation, elongation, and seed development. Plant Cell 15, 952-964.

Ruan, Y.L., et al., 2008. Expression of sucrose synthase in the developing endosperm is essential for early seed development in cotton. Functional Plant Biology 35, 382-393.

Sasaki, H., Ichimura, K., Imada, S., Yamaki, S., 2001. Sucrose synthase and sucrose phosphate synthase, but not acid invertase, are regulated by cold acclimation and deacclimation in cabbage seedling. J. Plant Physiol. 158, 847-852.

Schafer, W.E., Rohwer, J.M., Bothaf, F., 2004. Protein-level expression and localization of sucrose synthase in the Sugarcane culum. Plant Physiol. 121, 187-195.

Sebkova, V., Unger, C., Hardegger, M., Sturm, A., 1995. Biochemical physiological and molecular characterization of sucrose synthase from Daucus carota. Plant Physiol. $108,75-83$. 
Shi, J., Wang, H., Hazebrok, J., Ertl, D.S., Harp, T., 2005. The maize low-phytic acid three encodes a myo-inositol kinase that plays a role in phytic acid biosynthesis in development seed. Plant J. 42, 708-719.

Silvente, S., Camas, A., Lara, M., 2003. Heterogeneity of sucrose synthase genes in bean (Phaseolus vulgaris L.): evidence for a nodule-enhanced sucrose synthase gene. Journal Exp. Bot. 54, 749-755.

Smart, C.C., Flores, S., 1997. Overexpressing of D-myo-inositol-3-phosphate synthase leads to elevated levels of inositol in Arabidopsis. Plant Mol. Biol. 33, 811-820.

Smeekens, S., 2000. Sugar-induced signal transduction in plants. Plant Mol. Biol. 51, 49-81.

Stevenson, J.M., Perera, I.Y., Heilmann, I., Peresson, S., Boss, W.F., 2000. Inositol signaling and reproductive traits in soybean. Crop Sci. 43, 464-473.

Sturm, A., Lienhard, S., Schatt, S., Hardegger, M., 1999. Tissue-specific expression of two genes for sucrose synthase in carrot (Daucus carota L.). Plant Mol Biol. 39, 349-360.

Suzuki, M., Tanaka, K., Kuwano, M., Yoshida, K.T., 2007. Expression pattern of inosito phosphate-related enzymes in rice (Oryza sativa L.): implication for the phytic acid biosynthesis pathway. Gene 36016, 1-10.

Tang, G.Q., Sturm, A., 1999. Antisense expression of sucrose synthase in carrot (Daccus carota L.) affects growth rather than sucrose partitioning. Plant Mol. Biol. 41, 465-479.

Tanaze, K., Yamaki, S., 2000. Purification and characterization of two sucrose synthase isoforms from Japanese pear fruit. Plant Cell Physiol. 41, 408-414.

Turner, S., Somerville, C.R., 1997. Collapsed xylem phenotype of Arabidopsis identifies mutants deficient in cellulose deposition in the secondary cell wall. Plant Cell 9 , 689-701.

Tzafrir, I., et al., 2004. Identification of genes required for embryo development in Arabidopsis. Plant Physiol. 135, 1206-1220.

Wilcox, J., Premachandra, G., Young, K., Raboy, V., 2000. Isolation of high seed inorganic P, low-phytate soybean mutants. Crop Sci. 40, 1601-1605.

Zeng, Y., Wu, Y., Avigne, W.T., Koch, K.E., 1999. Rapid repression of maize invertases by low oxygen. Invertase sucrose synthase balance, sugar signaling potential, and seedling survival. Plant Physiol. 121, 599-608.

Zrenner, R., Salanoubat, M., Willmitzer, L., Sonnewald, U., 1995. Evidence of the crucial role of sucrose synthase for sink strength using transgenic potato plants (Solanum tuberisum L.). Plant J. 7, 97-107. 